\title{
Simulation Study on Semiconductor Laser Mode Locking Using Nonlinear Coupling in Multimode-Interference Waveguide Amplifiers
}

\author{
Jiann-Chang Lai, Yean-Woei Kiang, Member, IEEE, and C. C. Yang
}

\begin{abstract}
The feasibility of a passively mode-locked semiconductor laser with a multimode-interference (MMI) waveguide amplifier is numerically studied using a two-dimensional timedomain beam-propagation method. In an appropriately designed ring cavity, a pulse can be compressed from a few hundred picoseconds to several picoseconds, as a result of the interplay between linear coupling and gain saturation in the MMI waveguide amplifier. The asymptotically stable pulse peak position and pulsewidth imply the feasibility of passively mode-locking semiconductor lasers. The frequency chirping of the compressed pulse and the lateral distribution of the output beam are numerically analyzed in detail. Our simulations show that mode locking can be implemented within a fairly broad range of injection current and cavity alignment.
\end{abstract}

Index Terms - Gain saturation, mode locking, multimodeinterference, nonlinear coupling, semiconductor optical amplifier.

\section{INTRODUCTION}

$\mathbf{N}$ ONLINEAR coupling mode locking was first proposed for fiber lasers [1] and then for semiconductor lasers [2]. The basic principle of nonlinear coupling mode locking is to use the pulse compression mechanism in the process of nonlinear coupling in a directional coupler. Nonlinear coupling means that a low (high) power signal emerges from the cross (bar) output port of a directional coupler with one coupling length, which is made of a nonlinear material such as fiber and semiconductor [3]. This process results in pulse breakup in which the high-power part (leading to nonlinear optics) and the low-power part (leading to linear optics) of an input pulse split [4]. The pulse is thus compressed. In a directional coupler with Kerr nonlinearity, the compressed pulse is basically symmetric because of the fast optical nonlinearity. The scenarios of pulse compression are quite different in a directional coupler made of two parallel semiconductor optical amplifiers in which the used optical nonlinear refraction is induced by gain saturation. In this case, pulse compression comes from the mechanisms

Manuscript received February 16, 1999; revised July 23, 1999. This work was supported by the National Science Council, the Republic of China, under Grant NSC 87-2215-E-002-006, Grant NSC 87-2215-E-002-007, and Grant NSC 87-2215-E-002-009.

J.-C. Lai and Y.-W. Kiang are with the Department of Electrical Engineering and the Graduate Institute of Communication Engineering, National Taiwan University, Taipei, Taiwan, R.O.C.

C. C. Yang is with the Department of Electrical Engineering and the Graduate Institute of Electro-Optical Engineering, National Taiwan University, Taipei, Taiwan, R.O.C.

Publisher Item Identifier S 0018-9197(99)08554-1. of linear coupling of the leading tail and gain saturation for effectively suppressing the trailing tail. It has been numerically shown that the pulse compression mechanism could lead to effective passive mode locking of a semiconductor laser in which an amplifier directional coupler was placed inside a ring cavity and the oscillating signal circulated through the barport amplifier [2]. Meanwhile, it was observed that in some situations the output pulse from the cross port had a higher peak power and a shorter pulsewidth, compared with that from the bar port. Hence, if we use the cross port as the laser output coupler, the aforementioned situations lead to the possibility that the average output power is higher than the intracavity average power. The previous simulation results showed the feasibility of the passively mode-locked semiconductor laser based on nonlinear coupling.

An experimental study on semiconductor laser mode locking based on nonlinear coupling was also attempted [5]. In this experiment, for fabrication simplicity, a multimode-interference (MMI) waveguide semiconductor optical amplifier (SOA), instead of a directional coupling amplifier, was used. An MMI waveguide of two guided modes was also called a zero-gap directional coupler [6]. The evolution of nonlinear signals in such a device is similar in concept to that in a directional coupler. With an MMI waveguide of one-half beat length (usually called a coupling length), a weak signal shifts from one lateral side of the waveguide to the other. However, optical nonlinearity, if induced, can retain a part of the signal power on the input side. Nonlinear switching results with a pump-probe configuration in semiconductor MMI waveguides based on absorption saturation were reported [6], [7]. In this mechanism, absorption saturation implies variations of waveguide lateral carrier distribution. This carrier redistribution is equivalent to a refractive index variation. Such an optically induced refractive index variation leads to nonlinear coupling. In our study, a passive waveguide is replaced by an amplifier, in which gain saturation would occur. The gain saturation also induces refractive index change through the Kramers-Kronig relations and leads to nonlinear coupling, similar to the case of a passive waveguide. In the aforementioned experiment for implementing passively mode-locked semiconductor lasers, although the autocorrelation of the output signal was not measured yet due to the lower output power, the observations of the dependence of the pulse repetition rate on the cavity length and the broadening of the pulsed spectrum provided strong evidences of mode locking [5]. 
Although passive mode locking of semiconductor laser based on nonlinear coupling has been numerically demonstrated based on a simple model, many detailed and more practical considerations were not implemented. Meanwhile, the first attempt of an experiment was conducted with an MMI SOA. The nonlinear switching and hence mode-locking mechanisms in an MMI SOA are actually different from those in a directional coupler. For instance, the linear coupling (due to the interference of the guided modes) in an MMI structure is much stronger than that in a directional coupler. Therefore, it is necessary to conduct detailed studies, theoretically and numerically, on the passive mode-locking mechanism with an MMI SOA. In this paper, we present the results of such studies. In Section II, the theoretical formulations are described. The numerical algorithms for simulation are given in Section III. Included in Section IV are the simulation results. Finally, discussions and conclusions are given in Section V.

\section{THEORETICAL FORMULATIONS}

To investigate the mode-locked semiconductor lasers with MMI SOA's, we need to first analyze the propagation of an optical pulse in the key component-the MMI SOA. We consider an SOA with a ridge-loading waveguide structure shown in Fig. 1. The ridge width is $8 \mu \mathrm{m}$. Although our theoretical and numerical results are quite general for any laser epitaxial structure, the following calculations are based on the epitaxial structure as follows. Four periods of quantum-wells/barriers (10-nm GaAs for wells and 20-nm $\mathrm{Al}_{0.25} \mathrm{Ga}_{0.75}$ As for barriers) are sandwiched by 200 -nm-thick i- $\mathrm{Al}_{0.25} \mathrm{Ga}_{0.75} \mathrm{As}$ separate confinement layers on both sides to form the guiding layer of thickness $0.43 \mu \mathrm{m}$. The upper and lower cladding layers are composed of $\mathrm{p}$ - and n-type $\mathrm{Al}_{0.6} \mathrm{Ga}_{0.4}$ As. We use the effective index method [8] for calculating the effective refractive index of the waveguide. Based on the waveguide structure described above, we first analyze an effective slab waveguide of thickness $0.43 \mu \mathrm{m}$ (along the $x$ direction) and find that only one guided mode exists in the epitaxial growth direction. Then, the lateral or $y$ direction guidance is provided by an effective slab waveguide of thickness $8 \mu \mathrm{m}$ to ensure the existence of two propagating modes in this dimension. From this model, it is found that the effective refractive indices (relating to the field distribution in the lateral direction, i.e., the $y$ axis) are $n_{1}=3.401$ and $n_{2}=3.3995$ in the ridge-loading region and outside, respectively. The coordinate system of the waveguide is also shown in Fig. 1 in which the wave propagates along the $z$ axis.

\section{A. Equations for Optical Pulse Propagation in an MMI SOA}

Since the TE mode usually dominates in a semiconductor laser, we only consider this mode, i.e., the electric field of interest is assumed to be polarized in the $y$ direction as

$$
\vec{E}(\vec{r}, t)=E(y, z, t) \hat{y} .
$$

Because the wave field profile in the $x$ direction is essentially unchanged during propagation, we will ignore the dependence on the $x$ coordinate in our wave propagation equation. This implies that the considered electric field is an average result
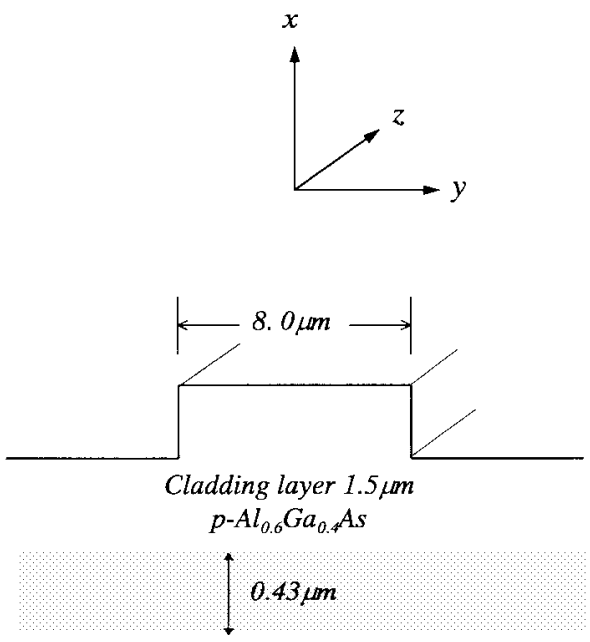

Cladding layer $1.5 \mu \mathrm{m}$ $n-A l_{0.6} G a_{0.4} A s$

Fig. 1. The MMI SOA with a ridge-loading waveguide structure.

over the distribution along the $x$ axis. Let us start with the wave equation for a stationary and local medium as

$$
\begin{array}{r}
\left(\frac{\partial^{2}}{\partial y^{2}}+\frac{\partial^{2}}{\partial z^{2}}\right) E(y, z, t)-\frac{1}{c^{2}} \frac{\partial^{2}}{\partial t^{2}} \int_{-\infty}^{t}\left[\varepsilon_{b}\left(y, z, t-t^{\prime}\right)\right. \\
\left.+\chi\left(y, z, t-t^{\prime}\right)\right] E\left(y, z, t^{\prime}\right) d t^{\prime}=0
\end{array}
$$

where $c$ is the speed of light in vacuum. We have purposely separated the dielectric constant into two parts: the susceptibility $\chi$ for the electron transitions related to the gain of the amplifier and the background dielectric constant $\varepsilon_{b}$.

By applying the slowly varying approximation with the complex amplitude $A(y, z, t)$ defined through

$$
E(y, z, t)=A(y, z, t) e^{j\left(\omega_{0} t-\beta_{0} z\right)}
$$

and assuming that the material response to the optical signal is instantaneous, (2) can be reduced to

$$
\begin{aligned}
\frac{\partial}{\partial z} A(y, z, t)= & \left\{\frac{c}{j 2 n_{0} \omega_{0}} \frac{\partial^{2}}{\partial y^{2}}+j \frac{n_{0} \omega_{0}}{2 c}\left(1-\frac{n_{b}^{2}}{n_{0}^{2}}\right)\right. \\
& -\left[\frac{n_{b}^{2}}{c n_{0}}+\frac{1}{c n_{0}} \chi(y, z, t)\right] \frac{\partial}{\partial t} \\
& -\frac{1}{c n_{0}} \frac{\partial \chi(y, z, t)}{\partial t}+\frac{\omega_{0}}{j 2 c n_{0}} \chi(y, z, t) \\
& \left.-\frac{1}{2} \rho_{\mathrm{int}}\right\} A(y, z, t) .
\end{aligned}
$$

Here, $\beta_{0}$ is a properly chosen propagation constant which can be expressed as $\beta_{0}=n_{0} k_{0}$ with $k_{0}=\omega_{0} / c$ being the vacuum wavenumber at the carrier frequency. Note that $n_{0}$ is chosen to be a reference refractive index, which is used for suppressing the fast-varying component of the wave field in the following calculations. Hence, it can be any value close to $n_{1}$ or $n_{2}$. The differences between the chosen $n_{0}$ and real effective indices of the waveguide modes will be absorbed by the slowly 
varying complex amplitude $A(y, z, t)$. Here, without loss of generality, we choose $n_{0}=n_{2}$ for numerical simplicity. Also, $n_{b}=\sqrt{\varepsilon_{b}}$ is the background refractive index. Meanwhile, we have introduced the optical loss in the SOA through adding the term with the loss constant $\rho_{\text {int }}$. Note that, in deriving (4), the second-order derivatives of the slowly varying envelope $A(y, z, t)$ with respect to $z$ and $t$ have been neglected under the paraxial approximation.

\section{B. Carrier-Density Rate Equation}

The relations among the electric field $E$, the susceptibility $\chi$, and the gain function in the MMI SOA are connected through the rate equation for carrier density $N$ as

$$
\frac{\partial N}{\partial t}=\frac{I}{q V}-\frac{N}{\tau_{c}}+D \nabla^{2} N-\frac{g}{\Gamma_{x}} \cdot \frac{|E|^{2}}{\hbar \omega_{0}} .
$$

Here, the four terms on the right-hand side account for carrier injection, carrier density relaxation, carrier diffusion, and stimulated recombination, respectively, where $I$ is the injection current, $q$ is the electron charge, $V$ is the active volume, $\tau_{c}$ is the carrier lifetime, $D$ is the carrier diffusion coefficient, $\hbar$ is Planck's constant divided by $2 \pi, \Gamma_{x}$ is the confinement factor in the $x$ direction, and $g$ is the gain constant which is a function of the carrier density. For simplicity in notations, we define the electric field $E$ such that $|E|^{2}$ stands for the optical power density or intensity $\left(\mathrm{W} / \mathrm{cm}^{2}\right)$.

Note that (5) is correct only when ultrafast intraband relaxation phenomena, such as spectral hole burning and carrier heating, can be neglected. Since we will be dealing with the pulses of at least several picoseconds, (5) is appropriate for describing carrier dynamics. Also note that we are concerned with the effects of carrier dynamics on pulse evolution in the time range from hundreds to a few picoseconds over a lateral dimension of around $8 \mu \mathrm{m}$. Within such a short time range, the effects of carrier diffusion can be neglected [9]. For instance, a lateral diffusion time constant at $1.4 \mathrm{~ns}$ for a scale size of $1.5 \mu \mathrm{m}$ was estimated in a GaAs-AlGaAs structure [6]. In our case, with the $8-\mu \mathrm{m}$ concerned lateral dimension, the diffusion time constant would exceed $10 \mathrm{~ns}$, which is much larger than the pulsewidth and carrier lifetime. Therefore, the diffusion factor is neglected in (5). However, under other conditions, the diffusion effect may deserve further investigation.

Although there are several more accurate models for the dependence of the gain constant $g$ on the carrier density $N$, without loss of generality, we consider the simple linear dependence [9] as

$$
g(y, z, t)=\Gamma_{x} a\left[N(y, z, t)-N_{0}\right]
$$

Here, $a$ is the differential gain coefficient and $N_{0}$ is the transparency carrier density.

By substituting (6) into (5), and using (3), we can obtain the equation for the gain constant $g(y, z, t)$

$$
\frac{\partial g(y, z, t)}{\partial t}=\frac{g_{0}-g(y, z, t)}{\tau_{c}}-\frac{|A(y, z, t)|^{2}}{E_{\mathrm{sat}} / \sigma} g(y, z, t)
$$

where $\sigma$ is the effective cross-sectional area of the waveguide, $E_{\text {sat }}$ is the saturation energy of the SOA, expressed as

$$
E_{\mathrm{sat}}=\frac{\hbar \omega_{0} \sigma}{a}
$$

and $g_{0}=\Gamma_{x} a N_{0}\left(I / I_{0}-1\right)$ is the small-signal gain constant with $I_{0}=q V N_{0} / \tau_{c}$ being the transparency current.

Based on the linear dependence assumed in (6), the susceptibility $\chi$ can be expressed in terms of the gain constant as

$$
\chi(y, z, t)=-\frac{c n_{0}}{\omega_{0}}(\alpha-j) g(y, z, t) .
$$

Here, the carrier-induced index change, responsible for self-phase modulation (SPM), is accounted for through the linewidth enhancement factor $\alpha$ [10]. It is due to this nonlinear refraction that the nonlinear coupling in an MMI SOA takes place.

With (9), (4) for wave propagation becomes

$$
\begin{aligned}
\frac{\partial}{\partial z} A(y, z, t)= & \left\{\frac{c}{j 2 n_{0} \omega_{0}} \frac{\partial^{2}}{\partial y^{2}}+j \frac{n_{0} \omega_{0}}{2 c}\left(1-\frac{n_{b}^{2}}{n_{0}^{2}}\right)\right. \\
& -\left[\frac{n_{b}^{2}}{c n_{0}}-\frac{1}{\omega_{0}}(\alpha-j) g(y, z, t)\right] \frac{\partial}{\partial t} \\
& +(\alpha-j) \frac{1}{\omega_{0}} \frac{\partial g(y, z, t)}{\partial t} \\
& +\frac{1}{2}(1+j \alpha) g(y, z, t) \\
& \left.-\frac{1}{2} \rho_{\text {int }}\right\} A(y, z, t) .
\end{aligned}
$$

Equations (7) and (10) are the equations to be numerically solved for understanding short-pulse propagation in an MMI SOA. For studying the mechanism of nonlinear coupling mode locking, the variations of the lateral wave-field distribution and the pulseshape are our major concern. The consideration of the lateral variation makes our numerical computations consume more effort. However, the results will provide new information previously not accessible.

\section{NumERICAL ALGORITHMS}

To demonstrate the numerical algorithm, we rewrite (10) as

$$
\frac{\partial}{\partial z} A(y, z, t)=\hat{O} A(y, z, t)
$$

where the nonlinear operator $\hat{O}$ is defined by

$$
\hat{O}=\hat{S}+\hat{T}+\hat{N}
$$

with

$$
\begin{aligned}
& \hat{S}=\frac{c}{j 2 n_{0} \omega_{0}} \frac{\partial^{2}}{\partial y^{2}}+j \frac{n_{0} \omega_{0}}{2 c}\left(1-\frac{n_{b}^{2}}{n_{0}^{2}}\right) \\
& \hat{T}=-\left[\frac{n_{b}^{2}}{c n_{0}}-\frac{1}{\omega_{0}}(\alpha-j) g(y, z, t)\right] \frac{\partial}{\partial t}
\end{aligned}
$$

and

$$
\hat{N}=(\alpha-j) \frac{1}{\omega_{0}} \frac{\partial g(y, z, t)}{\partial t}+\frac{1}{2}(1+j \alpha) g(y, z, t)-\frac{1}{2} \rho_{\mathrm{int}} .
$$


Note that $\hat{S}$ contains the effects of diffraction and lateral guiding, $\hat{T}$ takes care of the temporal variation of the electric field, and $\hat{N}$ accounts for the loss factor and the variations of gain in time and space.

For numerical computations, we employ a symmetrized split-step method, also known as the locally one-dimensional method [11], [12]. In this method, we divide longitudinally the MMI SOA into a series of steps with a step size $\Delta z$ and solve the equations step by step. In other words, we solve (11) for the pulse envelope $A(y, z+\Delta z, t)$ at $z+\Delta z$ in terms of $A(y, z, t)$ at $z$ through

$$
A(y, z+\Delta z, t)=e^{\hat{O} \Delta z} A(y, z, t) .
$$

Since $\hat{O}$ is the sum of several operators, we symmetrize the exponential such that the truncation error can be reduced to $O\left(\Delta z^{3}\right)$, according to Baker-Hausdorff's formula [11]. Here, (16) can be rewritten as

$$
\begin{aligned}
& A(y, z+\Delta z, t) \\
& \quad=e^{\hat{S} \Delta z / 2} e^{\hat{T} \Delta z / 2} e^{\hat{N} \Delta z} e^{\hat{T} \Delta z / 2} e^{\hat{S} \Delta z / 2} A(y, z, t) .
\end{aligned}
$$

The computational procedures are as follows. First, we focus our attention on the variation of the wave field along the $y$ direction with the execution of the operator $\hat{S}$. The execution of the exponential operator $\exp (\hat{S} \Delta z / 2)$ is carried out by the pseudospectral fast Fourier transform (FFT)-based technique [13]. This scheme is equivalent to solving the differential equation

$$
\frac{\partial A}{\partial z}=\hat{S} A, \quad z_{n}<z<z_{n}+\frac{\Delta z}{2} .
$$

The wave field is found at the half-step $\Delta z / 2$ by applying the following operation:

$$
A\left(y, z_{n}+\Delta z / 2, t\right)=\operatorname{PQPA}\left(y, z_{n}, t\right)
$$

where

$$
P=F^{-1}\left[\exp \left(j \frac{c \Delta z}{8 n_{0} \omega_{0}} k_{y}^{2}\right) F\right]
$$

and

$$
Q=\exp \left[j \frac{n_{0} \omega_{0} \Delta z}{4 c}\left(1-\frac{n_{b}^{2}}{n_{0}^{2}}\right)\right] .
$$

The operator $F$ means the Fourier transform and $F^{-1}$ means the inverse Fourier transform. The differential operator $\partial^{2} / \partial y^{2}$ can be easily replaced by $-k_{y}^{2}$ in the Fourier domain. Note that absorbers are needed (to be discussed later) at the lateral borders of the waveguide structure to avoid undesirable FFTinduced optical reflections.

Then, we consider the temporal variation of the wave field. The execution of the exponential operators $\exp (\hat{T} \Delta z / 2)$ and $\exp (\hat{N} \Delta z)$ is equivalent to solving the following differential equations:

$$
\frac{\partial A}{\partial z}=\hat{T} A, \quad z_{n}<z<z_{n}+\frac{\Delta z}{2}
$$

and

$$
\frac{\partial A}{\partial z}=\hat{N} A, \quad z_{n}<z<z_{n}+\Delta z .
$$

Here, the operators $\hat{T}$ and $\hat{N}$ are treated with the finite difference method as

$$
\begin{aligned}
\hat{T} A\left(y, z_{n}, t\right)= & -\left[\frac{n_{b}^{2}}{c n_{0}}-\frac{1}{\omega_{0}}(\alpha-j) g\left(y, z_{n}, t\right)\right] \\
& \cdot \frac{A\left(y, z_{n}, t+\Delta t\right)-A\left(y, z_{n}, t\right)}{\Delta t}
\end{aligned}
$$

and

$$
\begin{aligned}
\hat{N} A\left(y, z_{n}, t\right)= & {\left[(\alpha-j) \frac{1}{\omega_{0}} \frac{g\left(y, z_{n}, t+\Delta t\right)-g\left(y, z_{n}, t\right)}{\Delta t}\right.} \\
& \left.+\frac{1}{2}(1+j \alpha) g\left(y, z_{n}, t\right)-\frac{1}{2} \rho_{\text {int }}\right] A\left(y, z_{n}, t\right) .
\end{aligned}
$$

Obviously the solutions to (22) and (23) can be readily obtained.

Now let us summarize the procedures of numerical computations from $z=z_{n}$ to $z=z_{n+1}=z_{n}+\Delta z$. First, the gain equation (7) is solved by using the Runge-Kutta method [14] with the initial value $g_{0}$ and with the optical pulse field calculated at $z=z_{n}$. For optical field calculation, the pulse $A\left(y, z_{n}, t\right)$ first propagates over a distance $\Delta z / 2$ under the actions of the diffraction operator $\hat{S}$ and timederivative operator $\hat{T}$ using (19) and (22), respectively. At the midplane $z=z_{n}+\Delta z / 2$, the optical pulse is then subjected to the operator $\hat{N}$ that represents the nonlinear effect over the step $\Delta z$, using (23). Finally, the optical pulse propagates over the other $\Delta z / 2$ with the actions of the time-derivative operator $\hat{T}$ and diffraction operator $\hat{S}$, again using (22) and (19), respectively. This completes the operation from $z=z_{n}$ to $z=z_{n+1}$. Repeating this process gives the final lateral and temporal distributions of the optical pulse at the end of the MMI SOA.

As mentioned before, in considering the diffraction effect in (19), it is necessary to specify an absorbing boundary condition for the computational domain in $y$ to minimize the undesirable reflection from the numerical window. For this purpose, the lateral distribution of the refractive index is assumed to be

$$
\begin{aligned}
& n=n_{b}, \quad|y|<y_{\delta} \\
& n=n_{b}(1-j \delta), \quad y_{\delta}<|y|<y_{R}
\end{aligned}
$$

with the computational domain $|y|<y_{R}$ and the absorbing region $y_{\delta}<|y|<y_{R}$. The value of $\delta$ determines the absorption of the absorbing material. In order to minimize the error, we must place the absorber far from the waveguide so that the field amplitude is small enough to make this artificial loss tolerable. The appropriate location of the absorber depends on the particular problem we study and the accuracy we need.

For achieving numerical accuracy, we use the spatiotemporal moving frame consisting of $256 \times 256$ grid points. The step size $\Delta z$ is chosen to be $3 \mu \mathrm{m}$. The accuracy of our numerical algorithms is tested by two benchmarks: 1) check the "power loss" in a uniform medium and 2) compare the output pattern of a propagating Gaussian beam with the analytical solution. It is found that the numerical accuracy is satisfactory. Most of the computer calculation time is spent on the pseudospectral FFT process. The nonlinearity involved increases the calculation time significantly. About 15-25 min 
TABLE I

PARAMETERS FOR SiMULATIONS

\begin{tabular}{l|c|c|c}
\hline \multicolumn{1}{c|}{ Parameter } & Symbol & Value & Unit \\
\hline lateral width of simulation window & $W$ & 20 & $\mu m$ \\
\hline active waveguide length & $L_{a}$ & 0.66 & $\mathrm{~mm}$ \\
\hline passive waveguide length & $L_{p}$ & 1.32 & $\mathrm{~mm}$ \\
\hline active layer thickness & $d$ & 0.03 & $\mu \mathrm{m}$ \\
\hline coupling length & $L_{c}$ & 0.66 & $\mathrm{~mm}$ \\
\hline refractive index of guiding layer & $n_{1}$ & 3.401 & \\
\hline refractive index outside guiding layer & $n_{2}$ & 3.3995 & \\
\hline reference index & $n_{0}$ & 3.3995 & \\
\hline operating wavelength & $\lambda$ & 0.84 & $\mu \mathrm{m}$ \\
\hline saturation energy & $E_{s a t}$ & 5 & $\mathrm{pJ}$ \\
\hline linewidth enhancement factor & $\alpha$ & 6 & \\
\hline carrier lifetime & $\tau_{c}$ & 1 & $n \mathrm{~s}$ \\
\hline input energy of pulse & $E_{\text {in }}$ & 0.2 & $\mathrm{p.J}$ \\
\hline input pulse width & $\tau_{p}$ & 200 & $p \mathrm{~s}$ \\
\hline input beam waist & $y_{p}$ & 4 & $\mu m$ \\
\hline internal loss coefficient & $\rho_{\text {int }}$ & 50 & $\mathrm{~m}^{-1}$ \\
\hline & & &
\end{tabular}

are required on a $\mathrm{PC}$ (Pentium 133) for the calculation of pulse propagation along an MMI SOA $0.66 \mathrm{~mm}$ in length.

\section{SimUlation Results}

The parameter values of the MMI SOA used for simulation are listed in Table I. These values are collected either from the experiments [5] or from the literature [9]. The operating wavelength is at $\lambda=0.84 \mu \mathrm{m}$. Assume that the effective refractive index of the guiding layer is $n_{1}=3.401$ and that outside is $n_{2}=3.3995$. With this index distribution, if the lateral width of the loading ridge is $4 \mu \mathrm{m}$, only one guided mode exists. If the ridge width is $8 \mu \mathrm{m}$, two modes exist as mentioned before. Note that the internal loss coefficient used, $0.5 \mathrm{~cm}^{-1}$, is lower than the typical value in an SOA. However, this choice would not affect the physics of the phenomena discussed in this paper. It is expected that with an increased gain coefficient and loss coefficient, similar phenomena can be observed.

Linear coupling in the MMI SOA is due to the interference between the two guided modes. When a weak optical field is incident upon one half-side (called the bar port) of the MMI SOA from one end, the power is switched substantially to the other half-side (called the cross port) of the MMI SOA at the other end, if the length of the MMI SOA is equal to one coupling length.

From numerical simulations, it is found that the coupling length $L_{c}$ of our MMI SOA was $0.66 \mathrm{~mm}$. To see the nonlinear coupling phenomenon, we set the length $L_{a}$ of the MMI SOA to be one coupling length and let a pulsed Gaussian beam, as follows, be incident upon the bar-port side (positive $y$

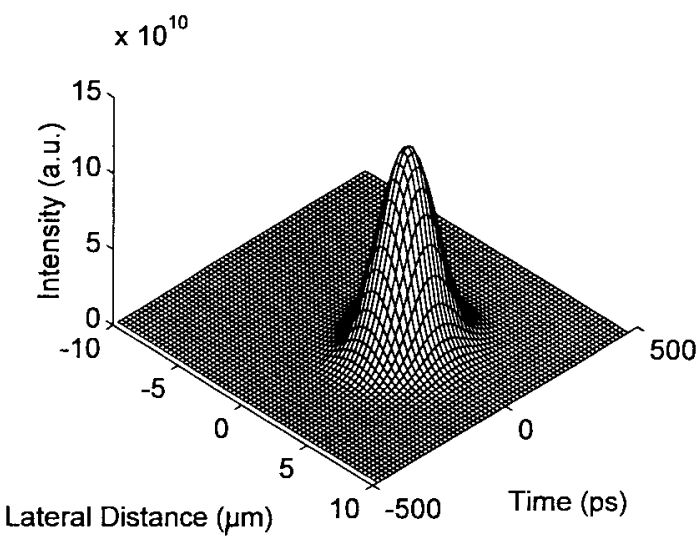

(a)

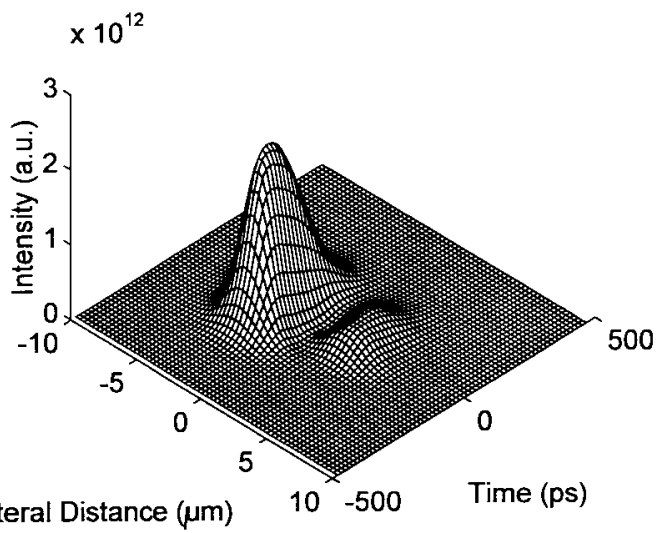

(b)

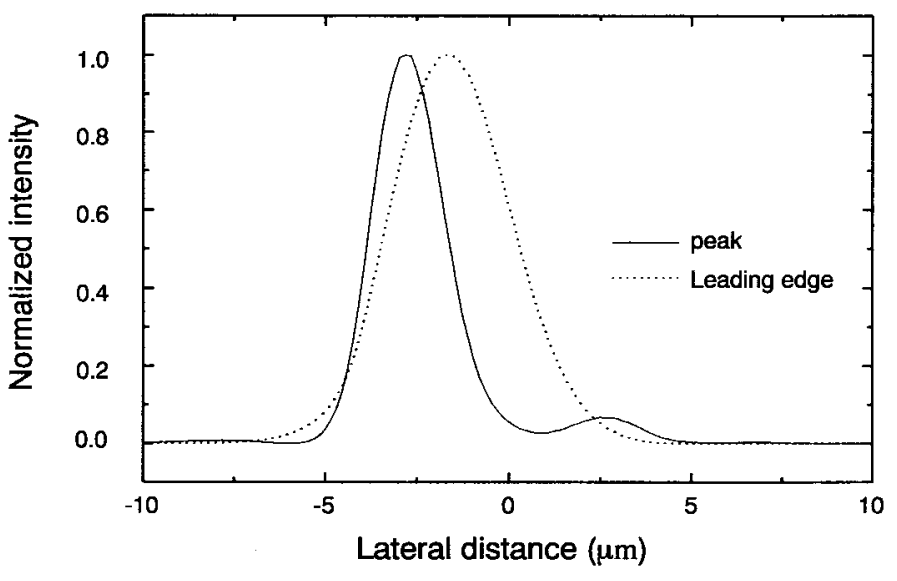

(c)

Fig. 2. (a) Space-time distribution of intensity for input pulse at $z=0$. (b) Space-time distribution of intensity for output pulse at $z=0.66 \mathrm{~mm}$. (c) Lateral patterns of normalized output intensity for the leading edge (dotted line) and the peak (solid line) of the pulse in the MMI SOA; $L_{a}=L_{c}$, $\alpha=6, G_{0}=30, \mathrm{~dB}, E_{\text {in }}=0.04 E_{\mathrm{sat}}$, and $\tau_{p}=200 \mathrm{ps}$.

coordinate) of the MMI SOA:

$$
A(y, z=0, t)=\sqrt{\frac{E_{\mathrm{in}}}{\tau_{0} y_{0} \pi}} e^{-\left(\left(y-y_{i}\right)^{2} / 2 y_{0}^{2}\right)} e^{-\left(t^{2} / 2 \tau_{0}^{2}\right)} .
$$

Here, the input energy $E_{\text {in }}$ is $0.2 \mathrm{pJ}$ (i.e., $E_{\text {in }}=0.04 E_{\text {sat }}$ ), the FWHM pulsewidth $\tau_{p}\left(=1.665 \tau_{0}\right)$ is $200 \mathrm{ps}$, the FWHM beam waist $y_{p}\left(=1.665 y_{0}\right)$ is $4 \mu \mathrm{m}$, and the lateral position of the beam center $y_{i}$ is $2 \mu \mathrm{m}$. Shown in Fig. 2(a) and (b) are the space-time distributions of intensity for input pulse 

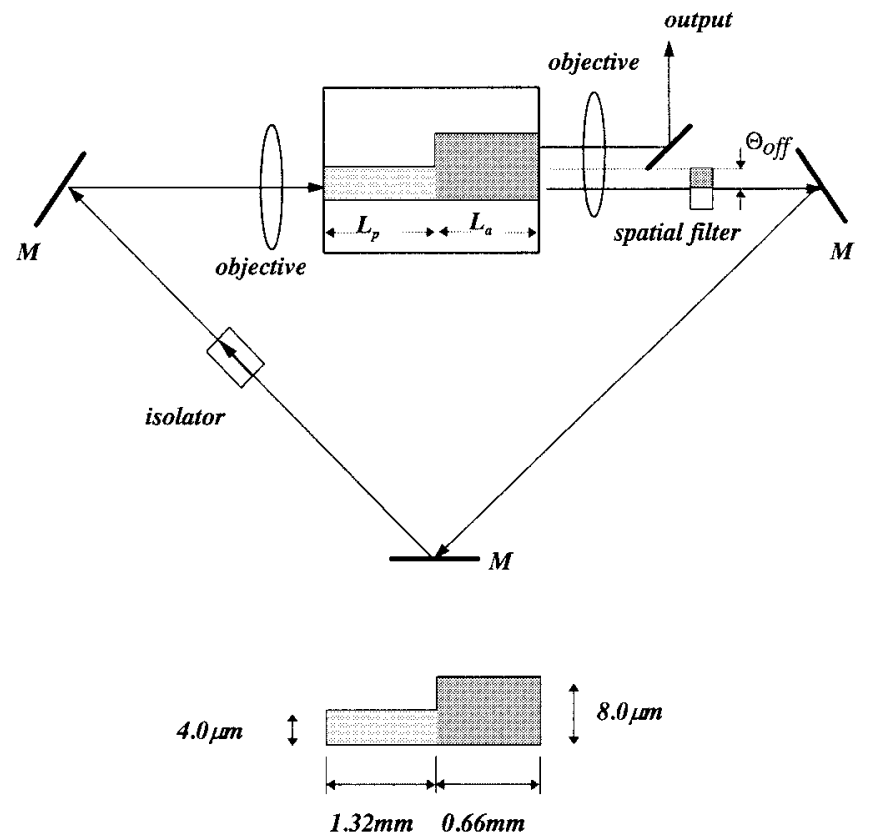

Fig. 3. Laser setup for nonlinear coupling mode locking based on an MMI SOA.

(at $z=0$ ) and output pulse (at $z=0.66 \mathrm{~mm}$ ), respectively, with the total unsaturated gain $G_{0}\left(=\exp \left(g_{0} L_{c}\right)\right)$ equal to $30 \mathrm{~dB}$. It can be seen that the leading edge of the pulse is switched to the cross-port side (negative $y$ coordinate), while some energy remains in the bar-port side near the peak of the pulse. To show this more clearly, the lateral distributions of the normalized intensity of the output pulse at two instants are plotted in Fig. 2(c). The dotted curve stands for the leading part of the pulse (at the local time $t=-300 \mathrm{ps}$ ), where the optical energy is coupled from the bar port to the cross port almost without distortion. The solid curve represents the peak of the pulse (at the local time $t=0$ ), where gain saturation in the MMI SOA is significant, causing some residual energy left in the bar port. If the part of the pulse emerging from the bar port is properly extracted and amplified, pulse compression can be achieved. This then leads to the idea of nonlinear coupling mode locking based on the MMI SOA.

The setup of the mode-locked semiconductor laser with an MMI SOA for our simulations is schematically shown in Fig. 3. The gain medium is the MMI SOA of which the length is chosen to be one coupling length. In front of it, we prepare a section of passive waveguide for trimming the lateral pattern of the input optical pulse before incidence upon the bar-port side of the MMI SOA. In practice, this arrangement is feasible with the quantum-well intermixing technique [15]. Three mirrors of $100 \%$ reflectivity are used to form a ring cavity so that the oscillating signal circulates through the bar-port side of the MMI SOA. Two objective lenses are used for collimating input and output optical beams. A spatial filter is also inserted for controlling the lateral pattern of the oscillating signal. It can block part of the bar-port optical energy by shadowing along the lateral direction an offset distance $\Theta_{\text {off }}$ measured from the center $(y=0)$ of the MMI SOA. We use the cross port as the laser output port and extract the output pulse with a tilted mirror. The length of the ring cavity is assumed to

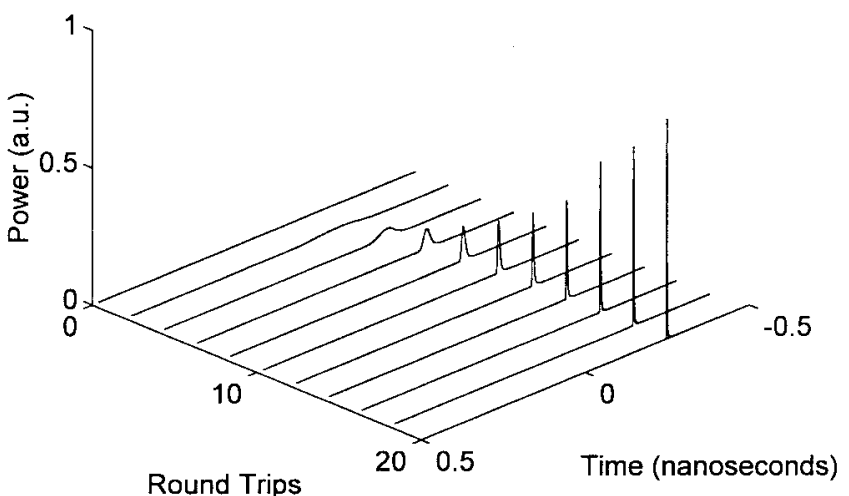

(a)

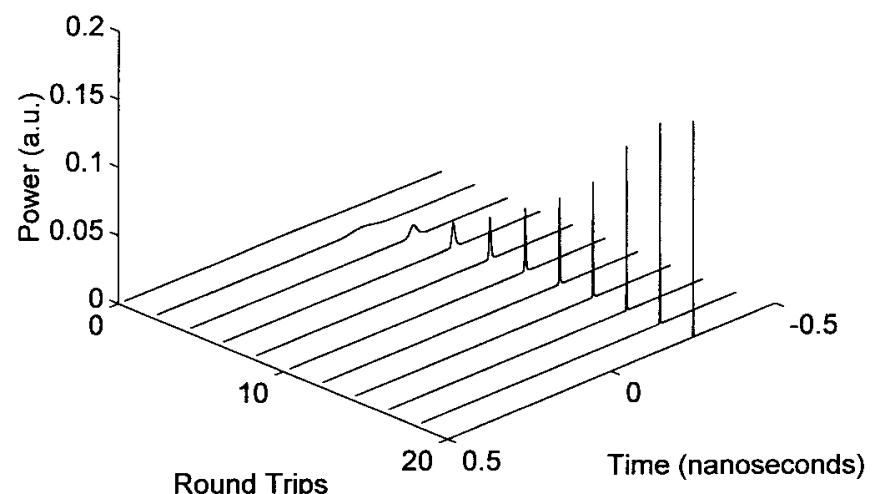

(b)

Fig. 4. Pulse evolution from the (a) cross and (b) bar ports in the MMI SOA. $L_{a}=L_{c}, \Theta_{\text {off }}=1.0 \mu \mathrm{m}, \alpha=6, G_{0}=25 \mathrm{~dB}, E_{\text {in }}=0.04 E_{\text {sat }}$, and $\tau_{p}=200 \mathrm{ps}$.

be much longer than the light speed times the carrier lifetime, so that the MMI SOA can recover from gain saturation when the circulated pulse reenters the MMI SOA. An isolator is used to guarantee one-way oscillation in the laser cavity. Meanwhile, we assume that both end facets of the MMI SOA are perfectly anti-reflection coated. Note that, in calculating the pulse evolution, we virtually connect the output of the spatial filter to the input of the passive waveguide section, disregarding the propagation effect (diffraction) in the cavity. The neglected diffraction effect may increase the loss level of the whole cavity. Because air cavity is assumed, no group velocity dispersion needs to be considered for this portion.

We start the simulation with a long Gaussian pulse (with FWHM pulsewidth $\tau_{p}=200 \mathrm{ps}$ ) to see whether the pulse can be compressed. The evolution of the pulse emerging from the cross and bar ports of the MMI SOA (with $G_{0}=25 \mathrm{~dB}$ ) is shown in Fig. 4(a) and (b), respectively. Here, the ordinate indicates the power obtained by integrating the intensity of the pulse over the lateral dimension for each port. We can see that the pulse is gradually compressed after a few round trips. Note that the peak power of the output pulse from the cross port is higher than that emerging from the bar port. This is similar to the result of nonlinear coupling mode locking based on a directional coupler [2]. The dynamic development of the compressed pulse is also shown in Fig. 5 by plotting the evolution of the FWHM pulsewidth and peak position during the first 20 round trips. The asymptotically steady values of 


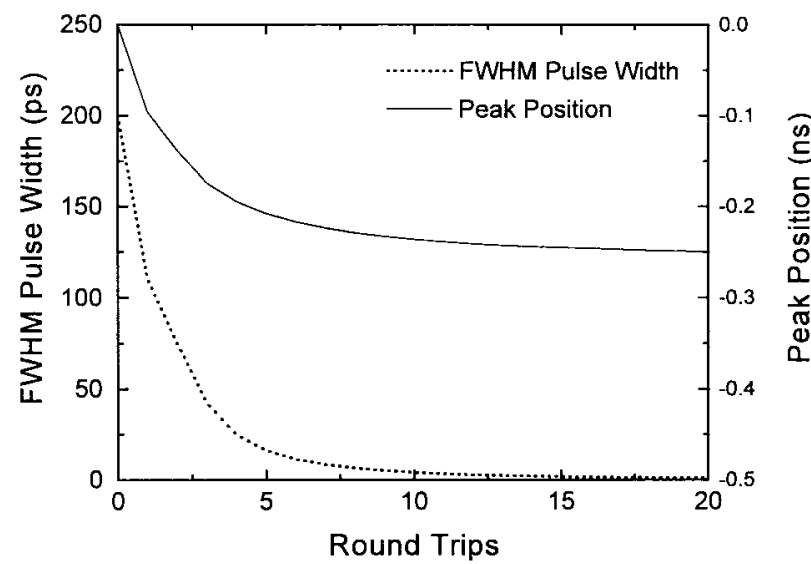

Fig. 5. Evolution of the FWHM pulsewidth (dotted line) and peak position (solid line) of the pulse from the bar port of the MMI SOA. $L_{a}=L_{c}, \Theta_{\text {off }}=1.0 \mu \mathrm{m}, \alpha=6, G_{0}=25 \mathrm{~dB}, E_{\text {in }}=0.04 E_{\text {sat }}$, and $\tau_{p}=200 \mathrm{ps}$.

the FWHM pulsewidth and peak position show the strong evidences of mode-locking feasibility.

The phenomenon of pulse compression is due to gain saturation as well as its induced nonlinear refraction in the MMI SOA. When the leading edge of the input pulse enters the bar-port side, the linear coupling mechanism transfers the weak part of the pulse into the cross port and makes the leading edge of the bar pulse become sharper. When the intense part of the optical pulse enters the SOA, gain saturation and the induced refractive-index change cause part of it to stay on the bar-port side. Moreover, the trailing part of the pulse experiences gain saturation and is suppressed. These mechanisms lead to effective pulse compression. Although only a short leading edge of the pulse is transferred into the cross port, after sufficient amplification, the cross pulse can be not only narrowed but also with a relatively higher intensity.

We vary the unsaturated gain over one coupling length $G_{0}=$ $\exp \left(g_{0} L_{c}\right)$ to examine its effect on the pulse compression. Illustrated in Fig. 6(a) and (b), representing the cross- and bar-port outputs, respectively, is the case of a low unsaturated gain at $G_{0}=15 \mathrm{~dB}$. Because the low unsaturated gain leads to insignificant gain saturation and hence negligible nonlinear coupling, most of the input pulse energy transfers into the cross port and very little is left in the ring cavity leading to vanishing oscillation signal. When we increase the unsaturated gain $G_{0}$ to $26 \mathrm{~dB}$, the pulse can be compressed, as shown in Fig. 7 . However, the stabilized peak position of the compressed pulse shifts a little bit forward in time when compared with the case in Fig. $4\left(G_{0}=25 \mathrm{~dB}\right)$. The reason for this phenomenon is that a higher unsaturated gain leads to a higher intracavity power and, hence, induces gain saturation earlier in time. Therefore, the circulated pulse and output pulse advance effectively. We also simulated the case with the unsaturated gain $G_{0}$ at $24 \mathrm{~dB}$ (not shown here) in which a compressed pulse with a stabilized peak position was observed. The above results show that the injection current needs to be well controlled within a range for providing an appropriate gain factor so that a steady modelocked pulse train can be obtained. From our simulations, this range of injection current can be fairly broad.

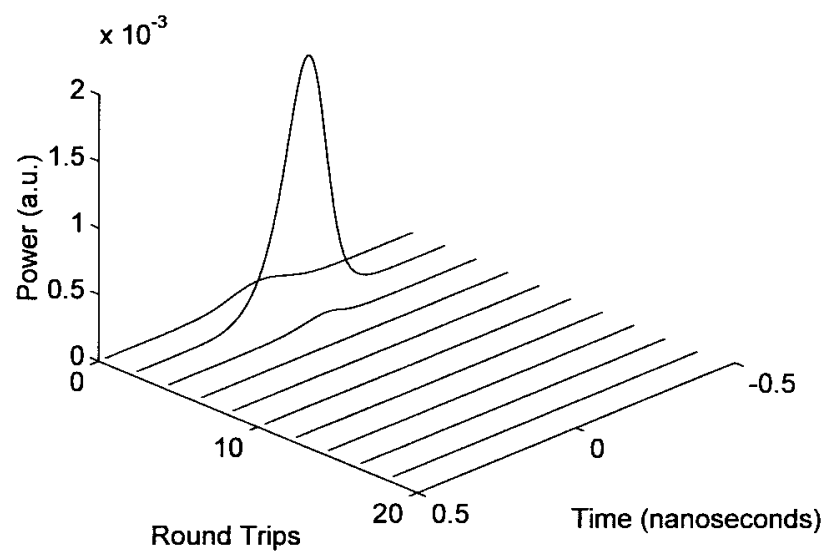

(a)

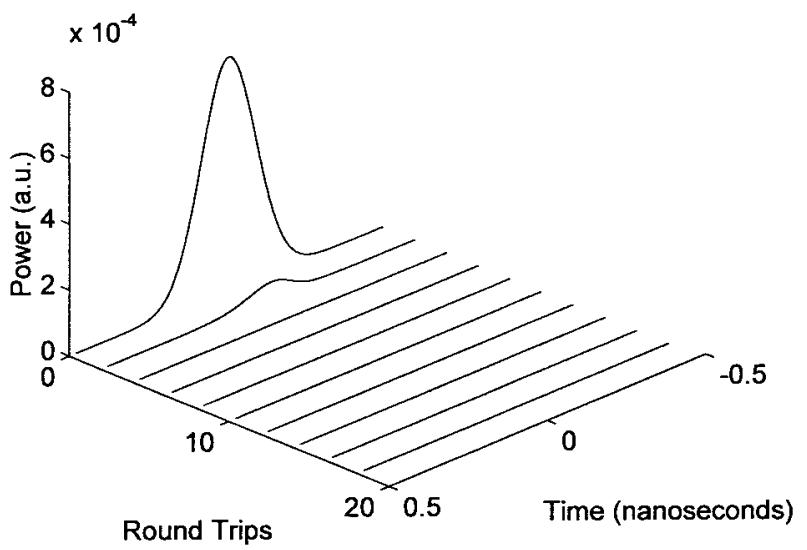

(b)

Fig. 6. Pulse evolution from the (a) cross and (b) bar ports in the MMI SOA. $G_{0}=15 \mathrm{~dB}, L_{a}=L_{c}, \Theta_{\text {off }}=1.0 \mu \mathrm{m}, \alpha=6, E_{\text {in }}=0.04 E_{\text {sat }}$, and $\tau_{p}=200 \mathrm{ps}$.

To understand the quality of the compressed pulse, the temporal variations of the normalized amplitudes (dotted curves) and phases (solid curves) of the complex amplitudes at five different lateral positions $[y=-4,-2.8,0,1.5$, and $3.5 \mu \mathrm{m}$, respectively, for (a)-(e)] after 20 round trips are plotted in Fig. 8. Here the simulation parameters are the same as those in Fig. 4. Note that the chosen positions $y=-2.8 \mu \mathrm{m}$ and $y=1.5 \mu \mathrm{m}$ are near peaks of the cross-port and bar-port, respectively, of the lateral distribution. From Fig. 8, one can find that frequency chirping is less prominent on the bar-port side, i.e., $y>0$. The essentially negative slope of phase within the pulse duration is consistent with the red shift trend of a pulse propagating in an SOA [9]. The small portions of positive-slope phase on the leading sides of the pulses when $y \leq 0$ represent small components of blue shift which can be attributed to spectral broadening of self-phase modulation. Plotted in Fig. 9 are the five spectra corresponding to the pulses in Fig. 8. Here the red shift of the spectrum is clearly seen. Note that there exists a peak near the input carrier frequency on the cross-port side $(y \leq 0)$. This part corresponds to the leading edge of the pulse which is only lightly affected by gain saturation.

To understand the output beam quality, in Fig. 10 we plot the lateral distributions of the normalized amplitudes (dotted curves) and phases (solid curves) of the 


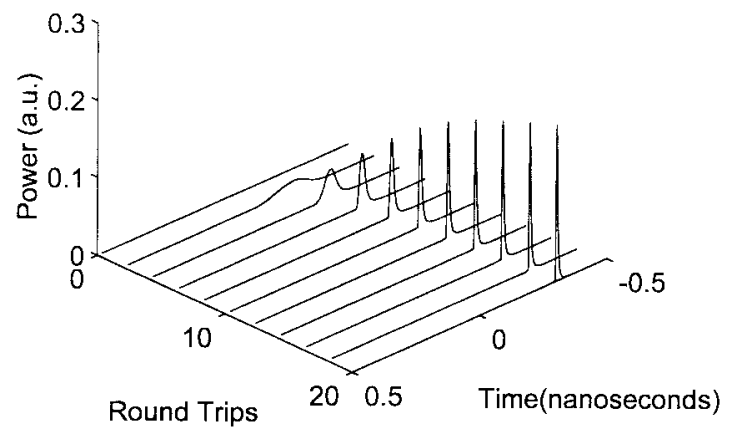

(a)

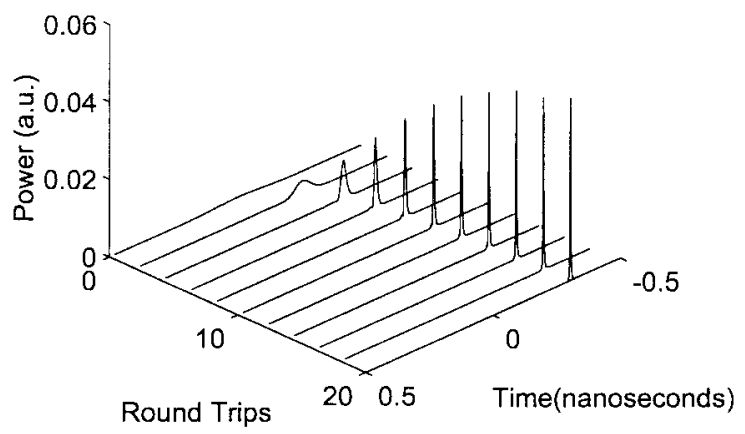

(b)

Fig. 7. Pulse evolution from the (a) cross and (b) bar ports in the MMI SOA. $G_{0}=26 \mathrm{~dB}, L_{a}=L_{c}, \Theta_{\text {off }}=1.0 \mu \mathrm{m}, \alpha=6, E_{\text {in }}=0.04 E_{\text {sat }}$, and $\tau_{p}=200 \mathrm{ps}$.

complex amplitudes at four different time instants $[t=$ $-0.25244,-0.25152,-0.25056$, and $-0.25018 \mathrm{~ns}$, respectively, for (a)-(d)] after 20 round trips. Fig. 10(b) and (c) here correspond to the temporal peaks in (d) and (c) of Fig. 8, respectively. The simulation parameters are still the same as those for Fig. 4. In all parts of Fig. 10, the major pulse power is distributed on the cross-port side $(y<0)$ with varying wavefronts. From (b) and (c), we can see that the output beam is expected to tilt toward the bar-port side $(y>0)$. In (a)-(c), particularly in (b), we can see the power left on the bar-port side. This part of the signal is essentially focusing after it leaves the SOA [see the solid curve of part (b)].

\section{DisCUSSIONS AND CONCLUSION}

With regard to the effect of filter offset $\Theta_{\text {off }}$, our simulations (not shown here) indicate that, if the filter offset is small, most of the bar-port energy, including weak and intense parts, is fed into the laser ring cavity. This then leads to the deterioration of pulse compression. On the other hand, if the pulse is passed through a window far from the center of the MMI SOA, i.e., with a large filter offset, it is easier to achieve pulse compression. This may result from better discrimination between the weak and intense portions of the pulse. Although the single-mode passive waveguide section at the input end also provides a filtering effect, diffraction may mix the weak and intense parts of the pulse during the propagation in the ring cavity, resulting in difficult discrimination between them. Therefore, the spatial filter near the output end is important in pulse compression. However, the drawback of this arrangement is that less energy remains inside the ring cavity and, hence, a higher injection current is required for maintaining laser operation.

We also move the lateral position of the single-mode passive waveguide which corresponds to the incident point on the lateral dimension of the MMI SOA. Numerical results (not shown here) reveal that mode locking can be achieved with several lateral positions of the passive waveguide, say, with shifting toward the cross-port side by $0.5,1$, or $1.5 \mu \mathrm{m}$. Therefore, our simulation results imply that a certain range of cavity alignment for achieving mode locking is allowed. This broad tolerance in the lateral direction should be helpful in implementing such a mode-locked laser.

In implementing a passively mode-locked laser, realistically the pulse grows from a noise burst rather than from an incident long pulse as our simulations. However, pulse compression into a steady state is the most important condition for passive mode-locking. Actually, the steady state of a passive mode-locked laser depends on the properties of the gain medium and cavity. It is independent of the initial pulse condition. Therefore, like those in literature, our study started with the simulation of pulse compression from an incident long pulse. During the pulse compression process, amplified spontaneous emission (ASE) may cause random fluctuations of the wave field and the induced gain saturation, and hence refractive index variation. Such random fluctuations would lead to random variations of width and position of pulse during pulse evolution. Although such effects would slow down the net effect of pulse compression, the passive mode-locking mechanism is still expected. Amplified spontaneous emission actually represents one of the effects causing instability of a mode-locked laser. The growth of a pulse from a noise burst and the effect of ASE on mode locking will be discussed in another publication.

In our laser cavity, we circulate the optical signal emerging from the bar-port side of the MMI. Therefore, the leading side of the pulse is clipped through linear coupling of the weak leading-edge part into the cross-port side. Meanwhile, the trailing side of the pulsed signal is effectively suppressed through gain saturation. The combined effect leads to pulse compression. In a real laser system, the pulse compression will be eventually balanced by pulse broadening due to groupvelocity dispersion (GVD). Hence, a steady-state pulse train can be obtained. However, in our simulations, we have not taken the effect of GVD into account yet. We can still obtain steady-state pulse trains. The results can be attributed to the following two reasons. First, as the pulse becomes narrower, its leading side becomes steeper. Therefore, the gain saturation effect occurs at an earlier time. This leads to a shorter linear-coupling part and hence a weaker clipping effect. Asymptotically, the pulse compression effect on the leading side disappears. The shorter linear-coupling part also explains the narrowing trend of the pulsed signal emerging from the cross-port side. Second, on the trailing side, the effective signal suppression due to gain saturation also diminishes as the pulsewidth becomes narrower. This phenomenon can be understood from (7). In this equation, because the first term on the right-hand side is much smaller than the second term, the temporal gain variation, i.e., $\partial g / \partial t$, is determined 


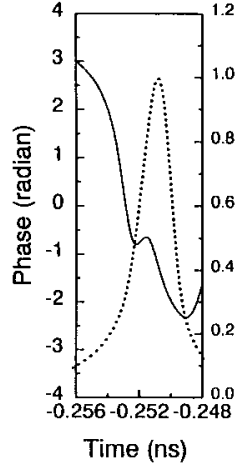

(a)

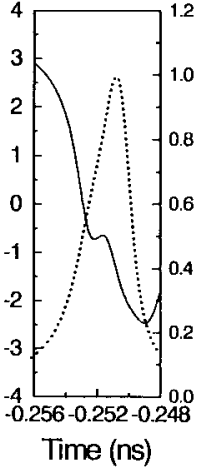

(b)

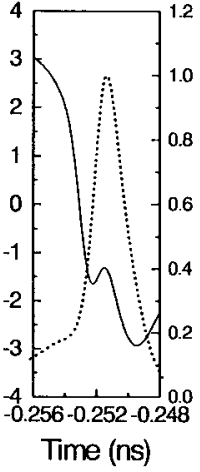

(c)

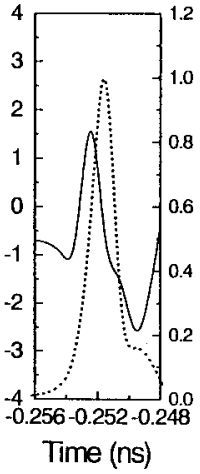

(d)

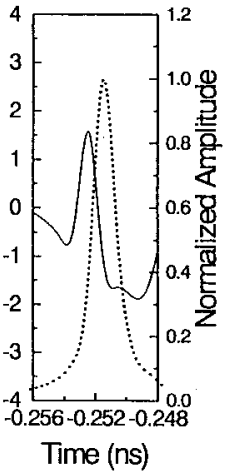

(e)

Fig. 8. Temporal variations of the normalized amplitudes (dotted curves) and phases (solid curves) of the complex amplitudes at five different lateral positions $[y=$ (a) -4, (b) -2.8, (c) 0 , (d) 1.5 , and (e) $3.5 \mu \mathrm{m}]$ after 20 round trips. $L_{a}=L_{c}, \Theta_{\text {off }}=1.0 \mu \mathrm{m}, \alpha=6, G_{0}=25 \mathrm{~dB}, E_{\text {in }}=0.04 E_{\text {sat }}$, and $\tau_{p}=200$ ps.

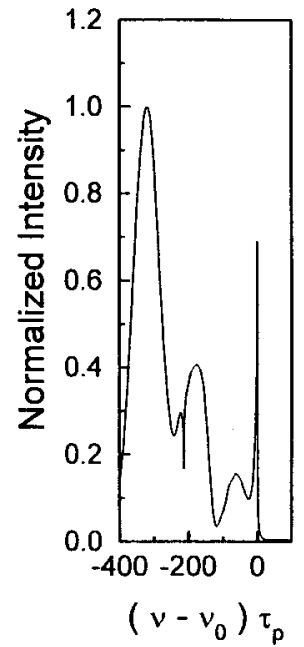

(a)

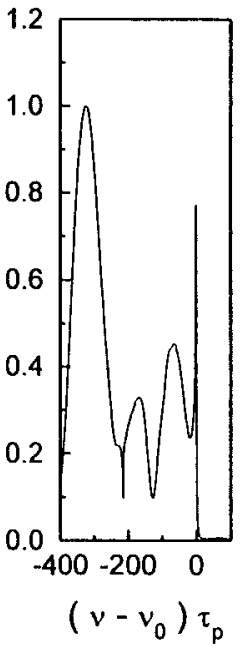

(b)

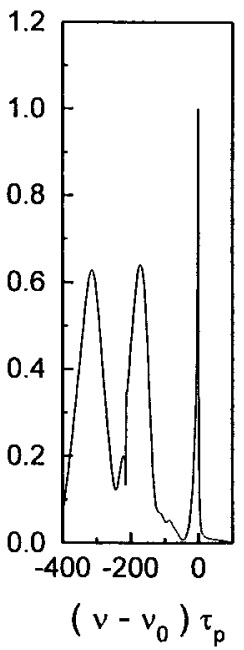

(c)

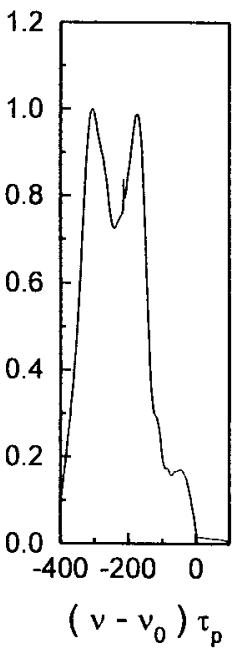

(d)

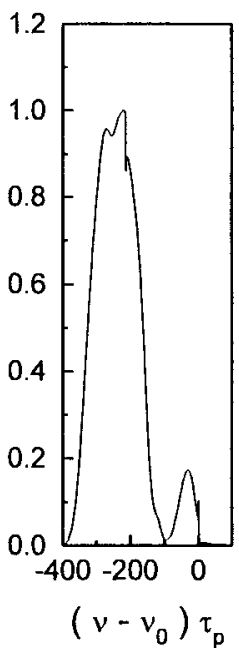

(e)

Fig. 9. Five spectra corresponding to the temporal distributions in Fig. 8.

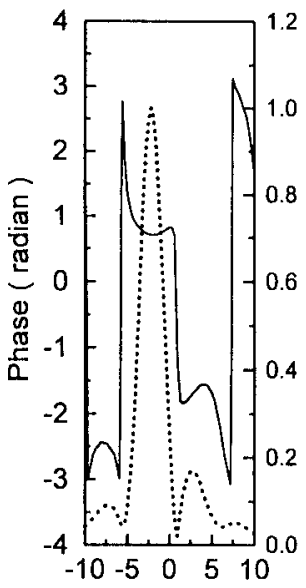

Lateral Distance ( $\mu \mathrm{m}$ )

(a)

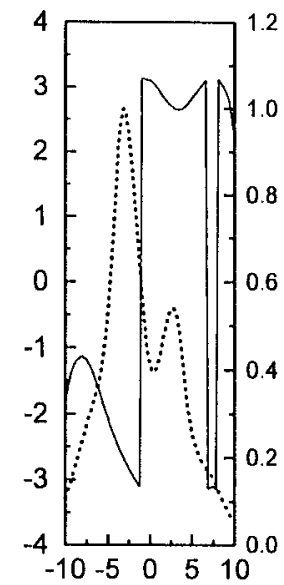

Lateral Distance $(\mu \mathrm{m})$

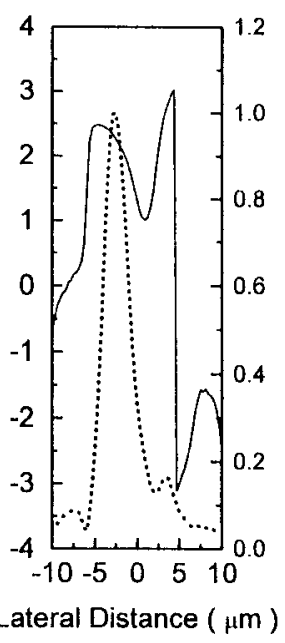

(c)

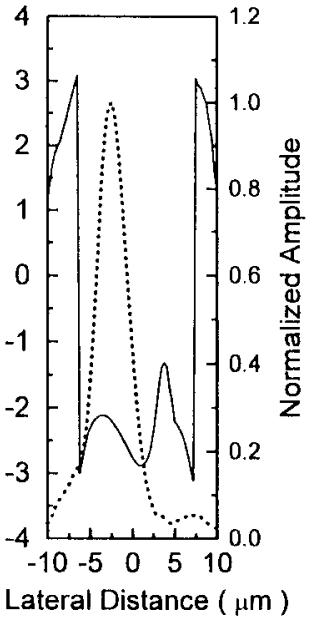

(d)

Fig. 10. Lateral distributions of the normalized amplitudes (dotted curves) and phases (solid curves) of the complex amplitudes at four different time instants $t=$ (a) -0.25244 , (b) -0.25152 , (c) -0.25056 , (d) $-0.25018 \mathrm{~ns}$ ] after 20 round trips. $L_{a}=L_{c}, \Theta_{\mathrm{off}}=1.0 \mu \mathrm{m}, \alpha=6, G_{0}=25$ $\mathrm{dB}, E_{\mathrm{in}}=0.04 E_{\text {sat }}$, and $\tau_{p}=200 \mathrm{ps}$.

predominantly by the second term on the right-hand side. Since the temporal gain variation determines the signal suppression on the trailing side, the pulse compression effect on the trailing side is controlled by the temporal variation of the second term, particularly after the pulse peak. When the pulse becomes quite narrow, the pulse intensity, i.e., $|A|^{2}$, drops fast after the peak, leading to a reduced magnitude of $\partial g / \partial t$. In other words, the pulse compression effect on the trailing side becomes 
less effective. The combination of the two reasons mentioned above explains the steady-state pulse train without considering the effect of GVD.

Regarding the observed stabilization of pulse position, we have the following explanation. Typically, a pulse shifts forward with the effect of gain saturation [9]. However, this forward shift counteracts against the clipping effect due to linear coupling. The balance between these two effects would lead to pulse position stabilization. In fact, as the pulse becomes narrower and its leading edge becomes steeper, the effects of both forward shift and clipping are gradually weakened.

Typically, in a mode-locked laser, the formation of a short pulse train may be caused by more than one pulse compression mechanism. Recently, the concept of self-bending was proposed to explain the passive mode locking of an external-cavity semiconductor laser [16]. The self-bending effect implies that the propagation direction of an optical signal depends on the power level when it emerges from the semiconductor optical amplifier. This self-bending effect is strongly related to the nonlinear coupling phenomenon discussed in this paper. The connection between these two mechanisms of mode locking is another issue of continuing study.

Pulse compression may start with a transient signal of nanoseconds in width. In this situation, it may be necessary to consider carrier diffusion. Carrier diffusion will blur the lateral carrier distribution, which is the key to the nonlinear refractive index variation and hence the nonlinear coupling mechanism. Therefore, carrier diffusion may have an effect of slowing down the pulse compression in our laser system.

In summary, using the beam-propagation method, we have numerically analyzed the operation of a nonlinear coupling mode-locked semiconductor laser with an MMI SOA. The nonlinear coupling mechanism is induced by gain saturation and associated refractive index change in the SOA. Attention has been particularly paid to the variation of the lateral wave field distribution, which is important in the design of the modelocked laser. It has been shown that a pulse of several hundred picoseconds can be compressed down to several picoseconds along the circulation of a ring cavity. This compression process implies the feasibility of passive mode locking. The frequency chirping and lateral distribution of the compressed pulsed beam have been numerically analyzed in detail. Our simulations also show that mode locking can be achieved in broad ranges of parameter values and with simple alignment. Future work also includes the simulation of the operation of such a mode-locked laser with a linear cavity.

\section{REFERENCES}

[1] H. G. Winful and D. T. Walton, "Passive mode locking through nonlinear coupling in a dual-core fiber laser," Opt. Lett., vol. 17, pp. $1688-1690,1992$

[2] C. W. Hsu and C. C. Yang, "Passive mode locking of semiconductor lasers based on nonlinear directional coupling," Opt. Lett., vol. 21, pp. 878-880, 1996.

[3] S. M. Jensen, "The nonlinear coherent coupler," IEEE J. Quantum Electron., vol. QE-18, pp. 1580-1583, 1982.

[4] S. R. Friberg, A. M. Weiner, Y. Silberberg, B. G. Sfez, and P. S. Smith, "Femtosecond switching in a dual-core-fiber nonlinear coupler," Opt. Lett., vol. 13, pp. 904-906, 1988.

[5] C. W. Lay, D. W. Huang, C. H. Lee, and C. C. Yang, "Mode-locking of a semiconductor laser with a multiple-mode interference waveguide," in
IEEE Lasers and Electro-Optics Soc. Annu. Meet., San Francisco, CA, Nov. 1997, Paper WZ2.

[6] P. LiKamWa, A. Miller, C. B. Park, J. S. Roberts, and P. N. Robson, "All-optical switching of picosecond pulses in a GaAs quantum well waveguide coupler," Appl. Phys. Lett., vol. 57, pp. 1846-1848, 1990.

[7] P. LiKamWa, A. Miller, J. S. Roberts, and P. N. Robson, " 130 ps recovery of all-optical switching in a GaAs multiquantum well directional coupler," Appl. Phys. Lett., vol. 58, pp. 2055-2057, 1991.

[8] T. Tamir, Ed., Integrated Optics. New York: Springer-Verlag, 1977.

[9] G. P. Agrawal and A. Olsson, "Self-phase modulation and spectral broadening of optical pulses in semiconductor laser amplifiers," IEEE J. Quantum Electron., vol. 25, pp. 2297-2305, 1989.

[10] M. Osinski and J. Buus, "Linewidth broadening factor in semiconductor lasers-An overview," IEEE J. Quantum Electron., vol. QE-23, pp. 9-23, 1987.

[11] S. Balsamo, F. Sartori, and I. Montrosset, "Dynamic beam propagation method for flared semiconductor power amplifiers," IEEE J. Select. Topics Quantum Electron., vol. 2, pp. 378-384, 1996.

[12] A. R. Mitchell, Computational Methods in Partial Differential Equations. New York: Wiley, 1969.

[13] M. D. Feit and J. A. Fleck, "Light propagation in graded-index optical fibers," Appl. Opt., vol. 17, pp. 3990-3998, 1978.

[14] W. H. Press, B. P. Flannery, S. A. Teuklsky, and W. T. Vetterling, Numerical Recipes: The Art of Scientific Computing. New York: Cambridge Univ., 1986.

[15] B. C. Qiu, A. C. Bryce, R. M. De La Rue, J. H. Marsh, "Monolithic integration in InGaAs-InGaAsP multiquantum-well structure using laser processing," IEEE Photon. Technol. Lett., vol. 10, pp. 769-771, 1998.

[16] P. Langlois and M. Piche, "Self-mode-locked semiconductor laser in a ring cavity," in 1998 Conf. Lasers and Electro-Optics Europe, Glasgow, U.K., Sept. 1998, Paper 20.20 CPD1.3.

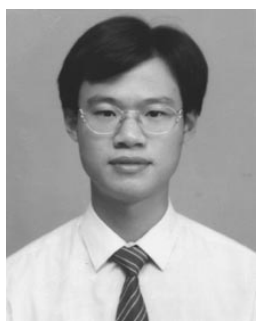

Jiann-Chang Lai was born in Taipei, Taiwan, R.O.C., in 1971. He received the M.S. degree in electrical engineering from National Taiwan University, Taipei, in 1998 .

His research interests concern numerical simulations of short optical pulse generation in semiconductor laser amplifiers. He is also interested in high-speed telecommunication networks. He is now an Engineer in the Long Distance \& Mobile Business Group, Chunghwa Telecom Corporation, Taipei.

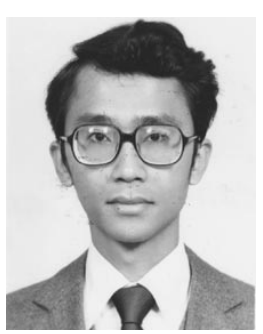

Yean-Woei Kiang (S'81-M'84) was born in Panchiao, Taiwan, R.O.C., in 1954. He received the B.S.E.E., M.S.E.E., and Ph.D. degrees from National Taiwan University, Taipei, in 1977, 1979 , and 1984 , respectively.

In 1979, he joined the Faculty of the Department of Electrical Engineering, National Taiwan University, where he is currently a Professor. His research interests include propagation, scattering, inverse scattering, and optoelectronics.

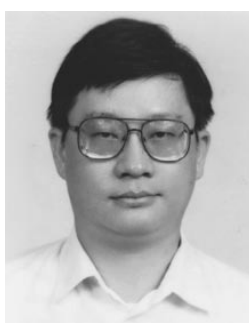

C. C. Yang was born in Cha-I, Taiwan, R.O.C., in 1954. He received the B.S. degree in electrical engineering from National Taiwan University, Taipei, in 1976, and the M.S. and Ph.D. degrees in electrical engineering from the University of Illinois at Urbana-Champaign in 1981 and 1984 respectively.

He then joined the Department of Electrical and Computer Engineering, The Pennsylvania State University, State College, as an Assistant Professor in 1984. He received his tenure and was promoted to Associate Professor in 1990. In 1993, he joined the Graduate Institute of Electro-Optical Engineering and the Department of Electrical Engineering, National Taiwan University, as a Professor. he has published more than 150 papers in technical journals and conferences. His research interests include ultrafast fiber and semiconductor optics, fabrication of fiber gratings and their applications, quantum-well intermixing and its applications, nonlinear optics, GaN-related materials and devices, and biophotonics. 